C-A/AP/\#114

October 2003

Booster Fault Study No. 16:

Deuteron Beam on the B6 Dump

L.A. Ahrens, K.A. Brown, C.J. Gardner

Brookhaven National Laboratory

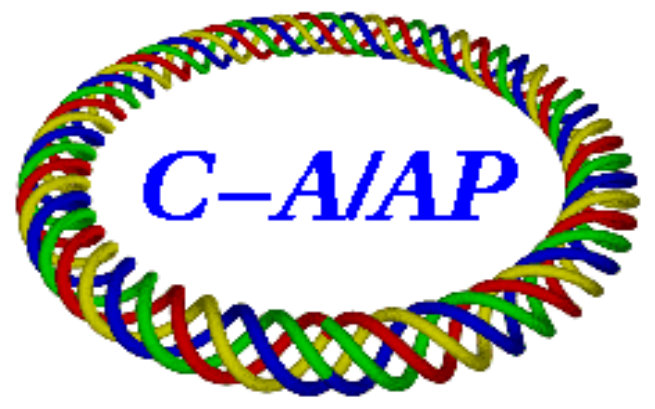

Collider-Accelerator Department Brookhaven National Laboratory

Upton, NY 11973 


\title{
Booster Fault Study No. 16: Deuteron Beam on the B6 Dump
}

\author{
L.A. Ahrens, K.A. Brown, and C.J. Gardner
}

October 8, 2003

Following is a report on the Booster fault study carried out on 22 January 2003. The original fault study plan is reproduced here followed by the actual study report.

\section{Study Plan}

\subsection{Goal}

The goal of this study is to produce a primary beam loss on the B6 Dump [1] and measure the resulting prompt radiation on the Booster berm directly over the dump and over the $\mathrm{C} 1$ dipole where three feed-through pipes come through the berm. Measurements also are to be made in the HEBT tunnel and in the TTB stub tunnel. The study is to be conducted in accordance with AGS OPM 9.1.9.

\subsection{Initial (non-fault) Beam Conditions}

1. The study is to be conducted with deuterons from Tandem or with protons from Linac.

2. The maximum kinetic energy is $1.0 \mathrm{GeV}$ per nucleon for deuterons and $2.0 \mathrm{GeV}$ for protons. The repetition period is at least 4 seconds.

3. The maximum intensity is $4 \times 10^{10}$ deuterons or $4 \times 10^{11}$ protons per Booster cycle. 


\subsection{Method}

1. Establish beam on a Booster cycle with flat top at the desired energy. Record flat top energy and repetition period.

2. The Booster RF should be turned off just after beam reaches flat top energy. Then, as the field increases (slightly) at the end of the flat top, beam will be lost on the radially inward side of the vacuum chamber.

3. Establish desired intensity with no loss on the flat top (until the field increases at the end). Record beam current transformer trace and ring loss monitor output throughout the Booster cycle.

4. Use the B6 dump bump [1] to produce a loss on the B6 dump as the field increases at the end of the flat top. Adjust the bump amplitude so that all beam on the flat top is lost on the dump.

5. Record beam current transformer trace and ring loss monitor output for the loss condition. Record output from Booster chipmunks. Record bump parameters.

6. Measure radiation levels on the Booster berm directly over the B6 dump and over the $\mathrm{C} 1$ dipole where three feed-through pipes come through the berm. Measure levels in the HEBT tunnel and TTB stub tunnel.

\subsection{Survey Locations}

Figure 1 shows a plan view of the region over the B6 dump extending from the B2 dipole to the $\mathrm{C} 4$ dipole. Figure 2 shows the cross-section of the Booster berm at the dump. Figure $\mathbf{3}$ shows a plan view of the region where the TTB line crosses the HEBT line and where the LTB line enters Booster. Here the TTB line crosses over the HEBT line near HEBT quadrupole NQ176. The part of the TTB tunnel downstream of the crossing point is called the TTB stub tunnel. The upstream face of the shielding through which the LTB line passes to enter the Booster ring is called the LTB headwall. A new gate called N132 has been installed in the HEBT tunnel. It is 44 feet upstream of NQ176. Refering to these drawings, the survey locations are: 
1. On Booster berm directly over the B6 dump. The vent shown in the figures can be used as a reference point to locate the point on the berm over the dump. The distance between this point and the vent is some 20 feet.

2. On the berm over the $\mathrm{C} 1$ dipole where the three feed-through pipes come through the berm. (This area is covered by a small steel-framed plywood-covered house.)

3. In the TTB stub tunnel at the downstream end. This area is to be surveyed only for the case of deuterons in Booster.

4. In the HEBT tunnel at the N132 gate. This area is to be surveyed only for the case of deuterons in Booster.

5. In the HEBT tunnel at the LTB headwall. This area is to be surveyed only for the case of deuterons in Booster.

All surveys are to be conducted with the HP1010 meter unless otherwise noted.

\subsection{Radiation Estimates}

\subsubsection{On the Berm over the B6 Dump}

To obtain an estimate of the radiation levels on the berm over the dump we assume a point loss on the dump and apply the formula

$$
H=\frac{1}{r^{2}}\left(1.6 \times 10^{-6}\right) L e^{-d / 117}
$$

given by Tesch [2]. Here $H$ is the dose rate (mrem/hour) on the berm directly above the loss point, $L$ is the loss rate $(\mathrm{GeV} / \mathrm{s}), d$ is the mass-density thickness $\left(\mathrm{g} / \mathrm{cm}^{2}\right)$ of the berm shielding, and $r$ is the distance (meters) from the loss point.

Refering to Figures $\mathbf{1}$ and $\mathbf{2}$, we see that on the berm directly over the B6 dump, the depth of the soil cover is $549 \mathrm{~cm}$ (18 feet) and the distance from the dump is $r=6.89 \mathrm{~m}$ (22.6 feet). Taking the density of the soil to be 1.8

$\mathrm{g} / \mathrm{cm}^{3}$, we have $d=549 \times 1.8=988 \mathrm{~g} / \mathrm{cm}^{2}$. For a point loss of $10^{11}$ protons per second at $2 \mathrm{GeV}$ kinetic energy, the dose rate on the berm would then be $1.5 \mathrm{mrem} /$ hour. For a point loss of $10^{10}$ deuterons per second at $1 \mathrm{GeV}$ kinetic energy per nucleon, the dose rate would be $0.15 \mathrm{mrem} /$ hour. 


\subsubsection{On the Berm over the C1 Dipole}

The three feed-through pipes that come through the berm over the $\mathrm{C} 1$ dipole are 8 inches in diameter and 18 feet long. They are spaced 2.5 feet apart. Two of the three have cables running through them; the third is empty and serves as a spare. Alan Stevens has done a calculation of the expected dose where the pipes come through the berm. He assumed two 10 inch diameter pipes with beam loss occuring directly beneath them in the $\mathrm{C} 1$ dipole. For protons with a kinetic energy of $2 \mathrm{GeV}$, the calculated dose is $5.17 \times 10^{-17}$ rem per proton. Thus, for a point loss of $10^{11}$ protons per second at $2 \mathrm{GeV}$ kinetic energy, the dose rate would be 18.6 mrem per hour. The dose rate for a point loss of $10^{10}$ deuterons per second at $1 \mathrm{GeV}$ kinetic energy per nucleon would be a factor of ten smaller.

\subsubsection{At the Downstream End of TTB Stub Tunnel}

J.W. Glenn has done a calculation of the expected dose rates at the downstream end of the TTB stub tunnel. He assumed a point loss of deuterons in the B8 dipole with 7 feet of sand and 2 feet of concrete between the loss point and the point of observation. For deuterons with a kinetic energy of $1 \mathrm{GeV}$ per nucleon and a loss of $1.2 \times 10^{12}$ deuterons per second, the calculated dose rate is 3.1 rem per hour. For a loss of $10^{10}$ deuterons per second the dose rate would be 26 mrem per hour.

\subsubsection{At the LTB Headwall}

J.W. Glenn has also done a calculation of the expected dose rates at the LTB headwall. He assummed a point loss of deuterons near the C3 quadrupole with 8 feet of light concrete between the loss point and the point of observation. For deuterons with a kinetic energy of $1 \mathrm{GeV}$ per nucleon and a loss of $1.2 \times 10^{12}$ deuterons per second, the calculated dose rate is 34 rem per hour. For a loss of $10^{10}$ deuterons per second the dose rate would be 283 mrem per hour. 


\section{Actual Study}

\subsection{Conditions}

Deuterons from Tandem with a kinetic energy of $17.4 / 2=8.7 \mathrm{MeV}$ per nucleon were injected into Booster and accelerated up onto the flat top of a Booster magnetic cycle. The flat top was $500 \mathrm{~ms}$ long and began $350 \mathrm{~ms}$ after BT0. The flat top field was 7121 Gauss. This gives a rigidity of 9.87 Tm (assuming radius of curvature $\rho=13.8656$ meters in the Booster dipoles) and a flat top kinetic energy of $814 \mathrm{MeV}$ per nucleon. At the end of the flat top, the field increased slightly before turning downward.

The magnetic cycle repetition period was $324 / 60=5.4$ seconds.

Under the fault conditions all beam loss occurred at the end of the flat top where the field increases. The loss was $2.2 \times 10^{10}$ deuterons per Booster cycle. With a repetition period of 5.4 seconds, this gives a loss rate of $0.4 \times 10^{10}$ deuterons per second.

\section{$2.2 \quad$ Loss Setup}

Just after beam reaches the flat top energy, the RF is switched off. Then, as the field increases at the end of the flat top, beam is lost on the radially inward side of the vacuum chamber. With a current of $100 \mathrm{~A}$ in the dump bump, most of the loss occurred in the region between the B6 and $\mathrm{C} 1$ dipoles. We refer to this as Fault Condition 1. With zero current in the dump bump, there were two distinct loss areas centered on the B4 and C4 dipoles. We refer to this as Fault Condition 2.

\subsection{Loss Measurements}

Beam loss was measured with the circulating beam current transformer and with the RLM (Ring Loss Monitor) system.

The current transformer was calibrated with a $5 \mathrm{~mA}$ calibration pulse which produced a $2.467 \mathrm{~V}$ pulse on an oscilliscope. Using the revolution period of $0.797 \mu$ s for deuterons with $814 \mathrm{MeV}$ kinetic energy per nucleon, one finds that a deuteron current of $5 \mathrm{~mA}$ corresponds to $2.49 \times 10^{10}$ deuterons in the Booster ring. The drop in the current transformer signal at the end of the flat top was $2.15 \mathrm{~V}$ for fault conditions 1 and 2. This gives a loss of $2.2 \times 10^{10}$ deuterons per Booster cycle. With a repetition period 
of 5.4 seconds, this gives a loss rate of $0.4 \times 10^{10}$ deuterons per second.

The RLM readings for fault conditions 1 and 2 are listed in Table 1. Here the window of observation was from 700 to $900 \mathrm{~ms}$ from BT0 which brackets the time when beam is lost at the end of the flat top.

Table 1: Booster RLM readings

\begin{tabular}{|c|c|c|}
\hline RLM & Fault 1 & Fault 2 \\
\hline B1 & 0 & 0 \\
B2 & 0 & 0 \\
B3 & 0 & 0 \\
B4 & 86 & 486 \\
B5 & 21 & 290 \\
B6 & 151 & 160 \\
B7 & 156 & 47 \\
B8 & 121 & 22 \\
\hline C1 & 137 & 14 \\
C2 & 87 & 140 \\
C3 & 56 & 272 \\
C4 & 194 & 485 \\
C5 & 52 & 107 \\
C6 & 96 & 41 \\
C7 & 60 & 14 \\
C8 & 11 & 4 \\
\hline
\end{tabular}

\subsection{Radiation Measurements}

\subsubsection{On the Berm over the B6 Dump}

For these measurements the HP1010 meter was placed on the ground over the B6 dump.

A dose rate of 0.025 mrem per hour was observed under fault condition 1 .

A dose rate of 0.033 mrem per hour was observed under fault condition 2 .

For a point loss (on the dump) of $10^{10}$ deuterons per second at $1 \mathrm{GeV}$ kinetic energy per nucleon, the predicted dose rate (on the berm over the dump) is $0.15 \mathrm{mrem} /$ hour. The predicted dose rate for a point loss of $0.4 \times 10^{10}$ deuterons per second at $814 \mathrm{MeV}$ kinetic energy per nucleon is 
then $0.4 \times 0.814 \times 0.15=0.049$ mrem per hour. This is consistent with the observed dose rates under the fault conditions.

\subsubsection{On the Berm over the C1 Dipole}

For these measurements the HP1010 meter was placed on the ground at the upstream end of the house that covers the three pipes that come through the berm over the $\mathrm{C} 1$ dipole. The meter was also placed in the cable tray that comes out of the downstream end of the house.

Under fault condition 1 , nothing ( $<0.025$ mrem per hour) was seen on the HP1010 meter.

Under fault condition 2, a dose rate of 0.025 mrem per hour was observed at the upstream end of the house.

For a point loss (in the $\mathrm{C} 1$ dipole) of $10^{10}$ deuterons per second at $1 \mathrm{GeV}$ kinetic energy per nucleon, the predicted dose rate (where the pipes come through the berm) is 1.9 mrem per hour. The predicted dose rate for a point loss of $0.4 \times 10^{10}$ deuterons per second at $814 \mathrm{MeV}$ kinetic energy per nucleon is then $0.4 \times 0.814 \times 1.9=0.62$ mrem per hour. This is 25 times higher than the observed dose rate.

\subsubsection{At the Downstream End of TTB Stub Tunnel}

These measurements were made with the E600 "rem ball" placed on the floor at the downstream end of the TTB stub tunnel. The measurements are summarized in Table 2. Here we see that the dose rates due to fault conditions 1 and 2 are 540 and $190 \mu$ rem per hour respectively.

For a point loss (in the $\mathrm{B} 8$ dipole) of $10^{10}$ deuterons per second at $1 \mathrm{GeV}$ kinetic energy per nucleon, the predicted dose rate is 26 mrem per hour. The predicted dose rate for a point loss of $0.4 \times 10^{10}$ deuterons per second at $814 \mathrm{MeV}$ kinetic energy per nucleon is then $0.4 \times 0.814 \times 26=8.5 \mathrm{mrem}$ per hour. This is 15 times higher than the observed dose rate.

\subsubsection{At the LTB Headwall}

Here the HP1010 meter was placed on the ground at the headwall. The measurements are summarized in Table 3 . Here we see that the dose rate due to fault condition 2 is 0.2 mrem per hour. This evidently is coming from the loss centered on the $\mathrm{C} 4$ dipole. 
Table 2: Radiation Levels in TTB Stub Tunnel

\begin{tabular}{|c|c|}
\hline Condition & $\frac{\text { Dose Rate }}{(\mu \mathrm{rem} / \mathrm{h})}$ \\
\hline Beam Off & 130 \\
\hline Beam On, No Fault & 130 \\
\hline Fault 1 & 670 \\
\hline Fault 2 & 320 \\
\hline
\end{tabular}

Table 3: Radiation Levels at LTB Headwall

\begin{tabular}{|c|c|}
\hline Condition & $\begin{array}{c}\text { Dose Rate } \\
(\mathrm{mrem} / \mathrm{h})\end{array}$ \\
\hline Beam Off & 1.2 \\
\hline Beam On, No Fault & 1.2 \\
\hline Fault 1 & 1.2 \\
\hline Fault 2 & 1.4 \\
\hline
\end{tabular}

For a point loss (near the C3 quadrupole) of $10^{10}$ deuterons per second at $1 \mathrm{GeV}$ kinetic energy per nucleon, the predicted dose rate is 283 mrem per hour. The predicted dose rate for a point loss of $0.4 \times 10^{10}$ deuterons per second at $814 \mathrm{MeV}$ kinetic energy per nucleon is then $0.4 \times 0.814 \times 283=92$ mrem per hour. This is much higher than the observed dose rate. 


\subsubsection{At N132 Gate}

For these measurements the HP1010 meter was placed on the floor near the N132 gate. The measurements are summarized in Table 4. Here we see that even with no beam, the dose rate is 10 mrem per hour. This apparently is due to local activation of the HEBT beampipe. The increase of 8 mrem per hour with beam on presumably is due to a local loss of deuteron beam in the nearby TTB line.

Table 4: Radiation Levels at N132 Gate

\begin{tabular}{|c|c|}
\hline Condition & $\frac{\text { Dose Rate }}{(\mathrm{mrem} / \mathrm{h})}$ \\
\hline Beam Off & 10 \\
\hline Beam On, No Fault & 18 \\
\hline Fault 1 & 18 \\
\hline Fault 2 & 15 \\
\hline
\end{tabular}

\section{References}

[1] C.J. Gardner, "The New Booster Dump and Dump Bumps", C-A/AP/Note 46, March 2001.

[2] K. Tesch, Health Physics, Vol. 44, No. 1, pp. 79-82, January, 1983. 


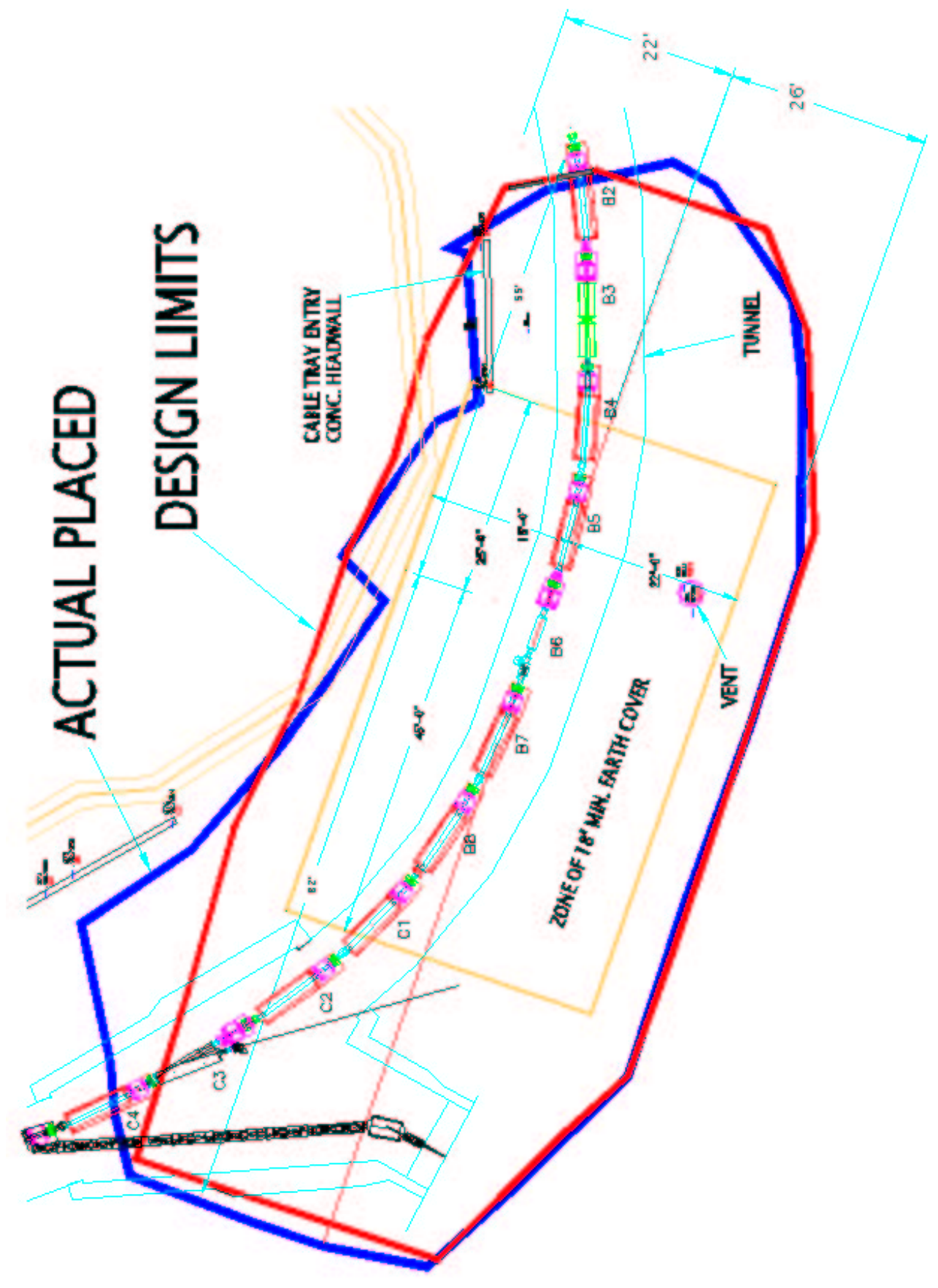

Figure 1: Plan view of the region over the Booster B6 Dump. 


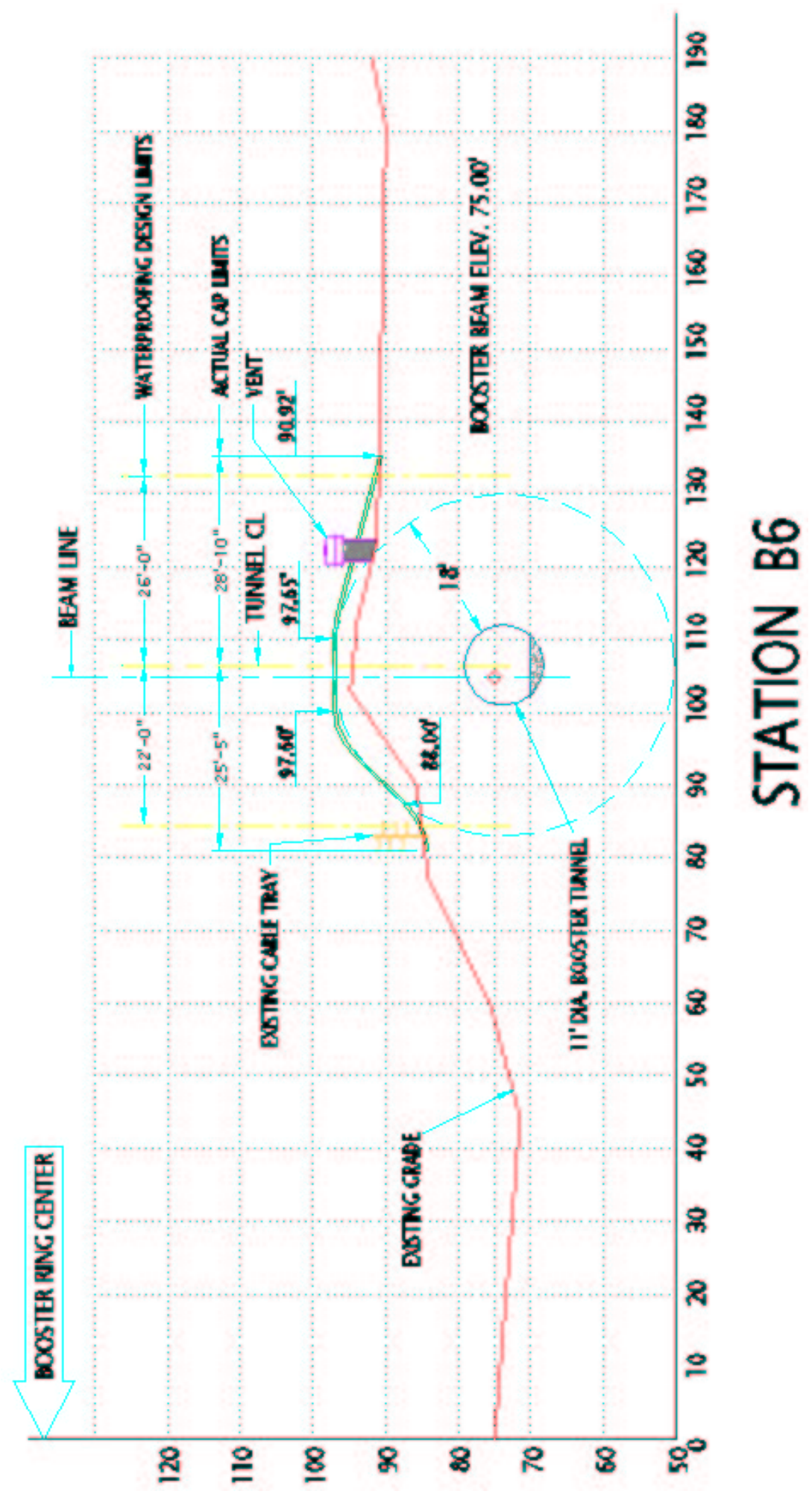

Figure 2: Cross-Section of Booster berm at the B6 Dump. 


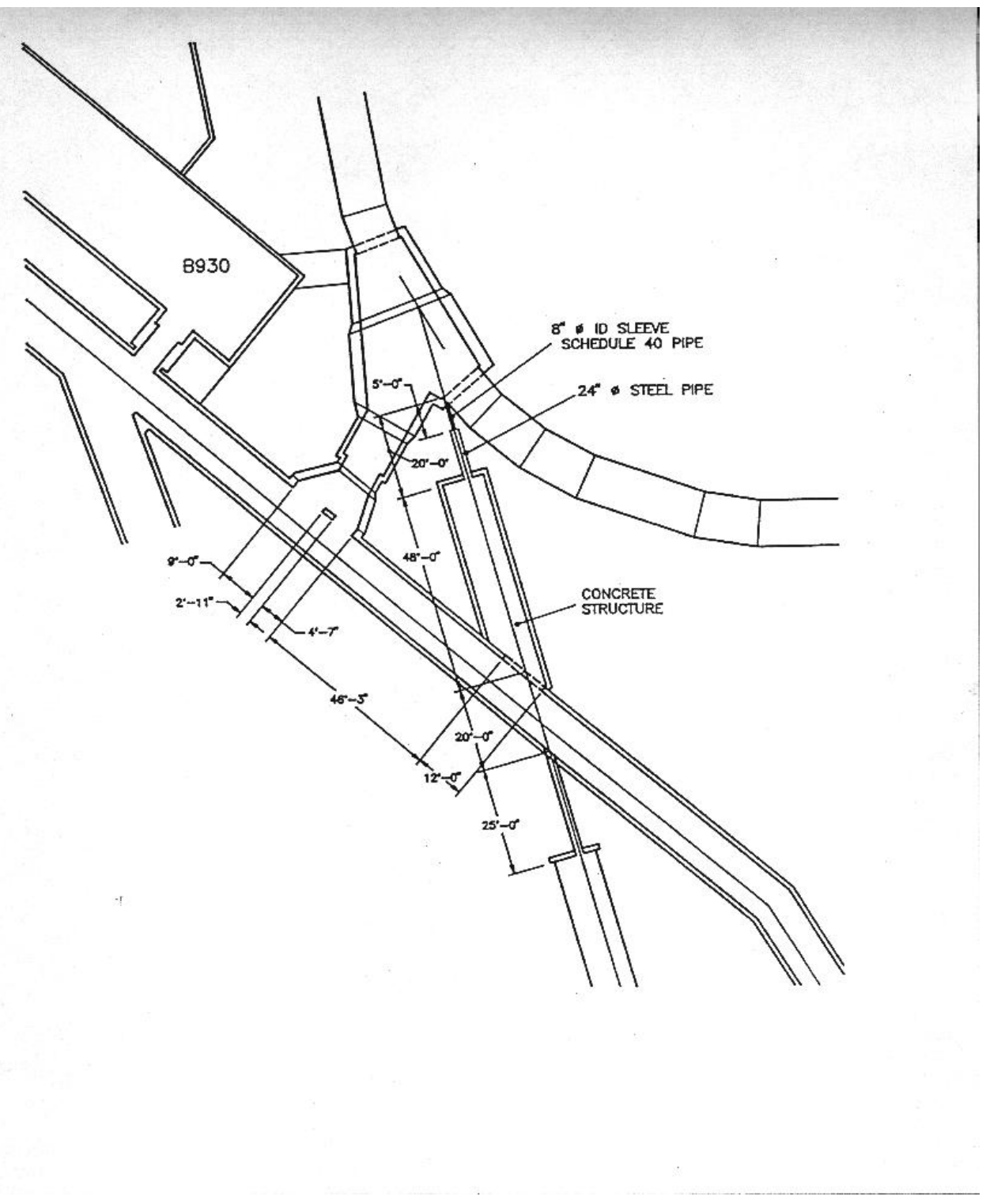

Figure 3: View of the region where the TTB line crosses the HEBT line and where the LTB line enters Booster. The part of the TTB tunnel downstream of the crossing point is the TTB stub tunnel. 